\title{
Evaluation of Arthroscopy Versus MRI in Anterior Cruciate Ligament Injuries
}

\author{
Mayurika Singh ${ }^{1}$, Yajuvendra Kumar Sharma ${ }^{2}$ \\ ${ }^{1}$ Assistant Professor, Department of Radiodiagnosis, M.L.N. Medical College, Allahabad, ${ }^{2}$ Senior Resident, Department of \\ Orthopaedics, S.N. Medical College, Agra, India
}

Corresponding author: Dr. Yajuvendra Kumar Sharma, Department of Orthopaedics, S.N. Medical College, Agra, India

DOI: http://dx.doi.org/10.21276/ijcmsr.2018.3.4.5

How to cite this article: Mayurika Singh, Yajuvendra Kumar Sharma. Evaluation of arthroscopy versus MRI in anterior cruciate ligament injuries. International Journal of Contemporary Medicine Surgery and Radiology. 2018;3(4):D18-D20.

\section{A B S T R A C T}

\section{Introduction:}

The knee is an important weight-bearing joint, capable of performing complex and extensive movements. The study endeavors to determine the role of MRI in diagnosing Anterior cruciate ligament injuries of the knee in comparison to diagnostic arthroscopy in Indian population.

Material and Methods: This prospective study was carried out in the Department of Radio diagnosis in collaboration with the Department of ORTHOPAEDICS (sports injury center) TERTIARY Hospital. 22 patients presenting with instability and pain of the knee joint and clinical suspicion of ACL injury included in the study. Two patients lost to follow-up hence, were excluded from the survey. These patients underwent MR imaging. Informed and written consent was taken from the patient before they included in the study. All 20 patients were subsequently followed up by arthroscopy/clinically examinations and correlated with findings on MR imaging.

Results: Sensitivity of MRI in the diagnosis of ACL injuries is high (90\%) however specificity is low. FSE sagittal T2 and coronal PD and STIR sequences were found to be more sensitive to the spectrum of cruciate ligament tears. STIR sequences were useful to evaluate associated bone contusions.

Conclusions: MRI has confirmed as the ideal approach for primary diagnosis of ACL lesions. It is noninvasive, fast, can be done on the outpatient basis and is free of complications as compared to diagnostic arthroscopy. But Arthroscopy still remains gold standard for ACL tear diagnosis.

Key words: Magnetic Resonance Imagine (MRI), Arthroscopy, Anterior Cruciate Ligament (ACL)

\section{INTRODUCTION}

Knee is frequently subjected to direct trauma due to its anatomical configuration and because it is the largest joint in the human body. ${ }^{1}$ Therefore it is one of the most affected joints by traumatic conditions.

Knee pain is a complaint in $20 \%$ of the general adult population and trauma; knee accounts second most common complaint. Sports-related activities account for approximately $60 \%$ of knee injuries producing ligament laxity. The incidence of knee injuries has increased in countries where sporting activities such as skiing, soccer, and baseball are in popularity. Pain and related symptoms may derive from damage to one or more of the soft tissues structures that stabilize and cushion the knee joint which include the ligaments, muscles, tendon, and menisci or from trauma to the bones forming the joint.

Most injuries to bones can easily diagnose on the plain $\mathrm{x}$-ray. However, soft tissues injuries of the knee are one of the most common and clinically challenging musculoskeletal disorders in patients presenting to the emergency department. Therefore establishing clear-cut diagnostic and therapeutic objectives for these injuries are important. Accurate and timely diagnosis increases the likelihood of fully restoring, regular and pain-free use of the affected knee. Misdiagnosis or mismanagement of damage to supporting structures of the knee may lead to chronic knee instability with subsequent development of degenerative joint disease and loss of knee function. In general, most injuries to knee arise from highenergy trauma such as motor vehicle accidents and sports. Therefore, patients tend to be young men. Ligamentous and meniscal Injuries are most likely in young and middle-aged adults whereas children and adolescents are more susceptible to osseous damage.

Magnetic resonance imaging has nowadays become the mainstay in the diagnosis of soft tissue injuries in and around the knee joint thus helping in alleviating patient's agony. The most significant advances in knee imaging have made in the realm of Magnetic Resonance Imagine (MR imaging). Since then, because of its improved Signal to Noise Ratio (SNR), higher resolution, reduced artifacts and shorter imaging times and improved accuracy of MRI, it has clearly emerged as the primary imaging tool in the workup of knee joint pathology. It has changed the traditional algorithm 
for knee joint pathology, particularly when cruciate ligament tears in cases of twisting injuries to the knee are suspected, and MRI has made it possible to look into the injured knee non-invasively thereby avoiding invasive procedures and further morbidity. Soft tissue discrimination with MR imaging is excellent and definite differences can make between cortex, marrow, ligaments, tendons, muscle, synovium, vascular and cartilaginous elements. ${ }^{2}$ Additional advantages of MR Imaging are multi-planar and thin section capabilities and the ability to evaluate subchondral bone and marrow. Kinematic and dynamic MR techniques provide an improved perspective for assessing the biomechanics of the patellofemoral joint and the standard functions of the ligamentous structures of the knee.

Arthroscopy has a dual advantage of being both diagnostic and therapeutic. But the use of anesthesia and requirement for hospital stay has become an important drawback in diagnostic ability of arthroscopy.

MRI being the new non-invasive screening modality in ligamentous injuries of the knee and hence can reduce the increasing load of diagnostic arthroscopy.

The study aimed to determine the role of MRI in diagnosing Anterior cruciate ligament injuries of the knee in comparison to diagnostic arthroscopy in Indian population.

\section{MATERIAL AND METHODS}

This prospective study was carried out in the Department of Radiodiagnosis in collaboration with the department of orthopedics (sports injury center) at Tertiary Hospital. 22 patients presenting with instability and pain of the knee joint and clinical suspicion of ACL injury included in the study. Two patients lost to follow-up hence, were excluded from the survey. These patients underwent MR imaging. Informed and written consent was taken from the patient before they included in the study. Patients age Between 15 to $45 \mathrm{yrs}$ with having a history of pain, swelling and instability following trauma and on clinical examination indicating ligamentous injuries were included in study. Patients Not consenting for imaging study as per protocol, unable to undergo MRI due to pacemakers aneurysm clip, metallic foreign body in or near the joint, having a contraindication to arthroscopy with previous history of knee surgery and having severe osteoarthritis, with a known history of joint disease e.g. rheumatoid arthritis etc. were excluded from study.

\section{STATISTICAL ANALYSIS}

Descriptive statistics like mean and percentages were calculated to interpret the results.

\section{RESULT}

A set of 20 patients (13 male and 7 female) presenting with instability and pain of the knee joint and clinical suspicion of ACL injury were included in the study. These patients underwent a physical examination, plain $\mathrm{x}$-ray MR imaging and subsequent arthroscopy / clinical follow-up.

Maximum patients belonged to age group between 15-25 years accounting of 11 patients (55\%). 26-35 years constituted the next major group. Youngest patient was 16 years old, and oldest was 40 years (table 1). Twisting injury was the

\begin{tabular}{|l|c|c|}
\hline Age (years) & No. of patients & Percentage \\
\hline $15-25$ & 11 & $55 \%$ \\
\hline $26-35$ & 7 & $35 \%$ \\
\hline $36-45$ & 2 & $10 \%$ \\
\hline Total & 20 & $100 \%$ \\
\hline \multicolumn{2}{|c|}{ Table-1: Distribution according to age } \\
\hline
\end{tabular}

\begin{tabular}{|l|c|}
\hline Mechanism of injury & No. of patients \\
\hline Twisting injury & 12 \\
\hline Anterior blow & 4 \\
\hline Fall & 2 \\
\hline Valgus & 1 \\
\hline Varus & 1 \\
\hline Total Table-2: According to mechanism of injury \\
\hline \multicolumn{2}{|c|}{20} \\
\hline
\end{tabular}

\begin{tabular}{|l|c|}
\hline Findings & No. of patients \\
\hline Medial meniscus tear & 2 \\
\hline Lateral meniscus tear & 1 \\
\hline Joint effusion & 15 \\
\hline Edema & 11 \\
\hline \multicolumn{2}{|c|}{ Table-3: Associated findings } \\
\hline
\end{tabular}

predominant mode of injury accounting for more than half of patients 12 (60\%). The anterior blow was the following common way of damage (Table 2). Sensitivity of MRI noted in diagnosing ACL injuries is of about 18 (90\%) however, specificity was only $27.5 \%$. Joint effusion was most common associated finding in the case of post-traumatic knee seen in $80 \%$. The second most common finding was marrow edema in surrounding bones noted in $55 \%$ cases (Table 3 ). While confirming the same with arthroscopy all patients had ACL injury.

\section{DISCUSSION}

In the age range of 15 to 45 with a mean age of 28 years. Maximum number patients 11 (55\%) were of the age group of $15-25$ years. It included 13 men and seven female patients who comprised of $65 \%$ and $35 \%$ respectively. It indicated males are seen to be more commonly affected rather female because of their increased incidence of physical activity in them. Our findings are seen to have discordance when compared to study conducted by Gwinn DE et $\mathrm{al}^{3}$ in 2007 which examined the relative incidence of anterior cruciate ligament injury in men and women at the United States Naval Academy, who found that women athletes had 3.96 times more chances of injuries as compared to their male counterparts. The study was conducted in active population and compared only the incidence of ACL injuries which is the reason of discordance between the findings. Our study includes all types of patients irrespective of the profession and has studied all kinds of ligament injuries. Anterior cruciate ligament tear noted in 2618 patients in MRI. 12(66.67\%) cases showed mid-substance tears; $4(22.22 \%)$ patients had tears at the femoral attachment and 2(11.11\%) at the tibial attachment. Bergquist et al. in 1991 in their study reported mid-substance tears as the commonest type (90\%). ${ }^{4}$ In 
1985 David A Turner et al. also in their published findings indicating mid-substance being the most common site of injury in 4 out of 6 cases. ${ }^{5,6}$ Abnormality of ACL contours on sagittal images is the most important initial sign indicative if its injury which noted in 12 cases. These findings are seen to be in concordance with G A Tung et al. in 1993 as quoted this conclusion to have the highest sensitivity and specificity with values of $96 \%$ (48 out of 50 examinations) and $94 \%$ (50 out of $53 \%$ ) on sagittal images. ${ }^{7}$ In secondary signs, bone contusion was the most common finding found in our study in 11 cases (55\%) followed by PCL abnormalities which were seen to collaborate with TR McCauley in 1994 who found bone contusion in 61 out 68 patients. ${ }^{8}$ However, E A Brandser et al. in 1996 found no difference in diagnostic performance when only initial signs used and when both primary and secondary signs used by comparing the area under receiving operating curve values being 0.80 for secondary and 0.93 for the primary. They found out that the secondary signs like the bone contusion, anterior translation of the tibia, and an uncovered posterior horn of the lateral meniscus to be the most useful for diagnosis. ${ }^{9}$ Mink et al. in $1988^{10}$ In tears of anterior cruciate ligament the sensitivity and specificity were found to be $96 \%$ and $25 \%$ respectively which is found partly corresponding to Fischer et al. in 1991 study as the precise nature of is low in our study. ${ }^{11}$ Study by Rezaei $Y$ et al. in 2007 also showed a low sensitivity and specificity $73 \%$ and $77 \%$ respectively. ${ }^{12}$ The diagnostic accuracy of MRI vs. clinical / arthroscopy was studied by and found results to be just above $90 \%$ in both the evaluations. The difference in the results could be due to small sample size.J K Lee et al. in 1988 found the sensitivity and specificity of MR imaging was $94 \%$ (17 of 18) and $100 \%$ on comparison with arthroscopy and clinical test. ${ }^{13}$ Similar results were found in our study with sensitivity of $90 \%$ and $100 \%$ on comparison with arthroscopy and clinical test.

\section{CONCLUSION}

The sensitivity of MRI in the diagnosis of ACL injuries is high (90\%) however, specificity is low. FSE sagittal T2 and coronal PD and STIR sequences were found to be more sensitive to the spectrum of cruciate ligament tears and coronal sequences for other ligament injuries. STIR sequences were useful to evaluate associated bone contusions. Arthroscopy is considered as" the gold standard" for the diagnosis of traumatic intraarticular knee lesion having an accuracy as high $100 \%$, however it is an invasive and expensive tool that requires hospitalization and regional or general anesthesia.

\section{REFRENCES}

1. Bruns W. Maftullli N. lower limb injuries in children in sports M ed. 2000; 19(4); 1-18.

2. Richard S. Snell. Clinical Anatomy for Medical Students. Fifth edition.

3. Cha JH, Chung HW, Kwon JW, Choi BK, Lee SH, Shin MJ2011 Longitudinal split of the posterior cruciate ligament: description of a new MR finding and evaluation of its potential clinical significance ClinRadiol. 2011;66(3):269-74.

4. Teng CD, Qiu QD. The value of MRI in diagnosis of ligament injuries of knee jointZhongguoGu Shang. 2010; 23(10):755-8.

5. Berguist TH. Magnetic resonance techniques in musculoskeletal diseases. Rheum clinNorth AM 1991; 17(3):599-615.

6. Kwanseop Lee,Marilyn J. Siegel, Debra M. Lau, Charles F. Hildebolt, and Matthew J. Matava. Anterior Cruciate Ligament Tears: MR Imaging-based Diagnosis in a Pediatric Population 1999;213(5):697-704.

7. G A Tung, L M Davis, M E Wiggins and P D Fadale. Tears of the anterior cruciate ligament: primary and secondary signs at MR imaging. Radiology 1993;3(6):188-90.

8. TR McCauley, M Moses, R Kier,JK Lynch,JW Barton, P Jokl. MR Diagnosis of tears of anterior cruciate ligament of the knee: importance of ancillary findings. Am. J. Roentgenol., 1994; 162(3): 115-119.

9. EA Brandser, MA Riley, KS Berbaum, GY el-Khoury and DL Bennett. MR imaging of anterior cruciate ligament injury: independent value of primary and secondary signs.American Journal of Roentgenology, 1996;167(6):121-126.

10. Mink JH, Levy T, Crues JV, Tears of the ACL and menisci of the knee: MR evaluation. Radiology 1988: 167(2): 769-774.

11. Fischer SP, Fox JM, Del pizzow et al. Accuracy of diagnosis from magnetic resonance imaging of the knee: A multi centre analysis of one thousand and fourteen patients. J Bone joint surg (AM) 1991; 73(5): 2-10.

12. Rezaei Y, Rahim nia A, Mirmohamad S M, Vaziri K, Fakhrejahani F. Sensitivity and specificity of MRI and Arthroscopy in knee joint injuries. Tehran university medical journal 2007;65(9):47-52.

13. J K Lee, L Yao, C T Phelps, C R Wirth, J Czajka, and J Lozman Anterior cruciate ligament tears: MR imaging compared with arthroscopyand clinical tests. Radiology March 1988;166(5):861-864.

\section{Source of Support: Nil; Conflict of Interest: None}

Submitted: 05-10-2018; Accepted: 30-10-2018; Published online: 06-11-2018 